\title{
Redirecting cardiac growth mechanisms for therapeutic regeneration
}

\author{
Ravi Karra ${ }^{1}$ and Kenneth D. Poss² \\ 'Department of Medicine and 'Department of Cell Biology, Duke University Medical Center, Durham, North Carolina, USA.
}

\begin{abstract}
Heart failure is a major source of morbidity and mortality. Replacing lost myocardium with new tissue is a major goal of regenerative medicine. Unlike adult mammals, zebrafish and neonatal mice are capable of heart regeneration following cardiac injury. In both contexts, the regenerative program echoes molecular and cellular events that occur during cardiac development and morphogenesis, notably muscle creation through division of cardiomyocytes. Based on studies over the past decade, it is now accepted that the adult mammalian heart undergoes a low grade of cardiomyocyte turnover. Recent data suggest that this cardiomyocyte turnover can be augmented in the adult mammalian heart by redeployment of developmental factors. These findings and others suggest that stimulating endogenous regenerative responses can emerge as a therapeutic strategy for human cardiovascular disease.
\end{abstract}

\section{Introduction}

Despite advances in the prevention and treatment of cardiovascular disease, heart failure remains a worldwide epidemic. For the 38 million patients with heart failure worldwide, the prognosis is grim: 5 -year mortality is approximately $50 \%$, a rate comparable to many cancers (1). Unfortunately, for patients with advanced disease, therapeutic options are limited. Cardiac transplantation is constrained by the availability of donor organs, and mechanical assist devices are associated with significant morbidity. Alternative approaches to treat heart failure are needed.

Heart failure often results from cardiomyocyte loss due to ischemic heart disease or by apoptosis (2). Heart regeneration is a relatively new and exciting field aimed to replace lost or dysfunctional cardiomyocytes. Current strategies for heart regeneration include reprogramming of scar-contributing cells to cardiac muscle-like cells, engineering cardiac tissue patches, and stimulating endogenous cardiomyocyte proliferation by adding factors or exogenous cells. While initial discoveries are promising, a better understanding of cardiac growth will provide needed context. Here, we will focus on lessons learned about cardiac growth from fish to humans and discuss the implications for achieving therapeutic heart regeneration.

\section{Modes of cardiac growth through development}

Although the 2-chambered zebrafish heart and the 4-chambered mammalian heart appear grossly different, mechanisms for cardiac development are highly conserved from fish to man. Indeed, zebrafish have proven to be a valuable tool for studying cardiac specification and chamber morphogenesis (3). In both zebrafish and mammals, initial cardiogenesis occurs by specification of uncommitted progenitor cells (Figure 1A and ref. 4). Combinatorial effects of local BMP, activin/nodal, FGF, Wnt, and retinoic acid sig-

Conflict of interest: The authors have declared that no conflict of interest exists. Reference information: J Clin Invest. 2017;127(2):427-436.

https://doi.org/10.1172/JCl89786. naling gradients direct and restrict the addition of cardiac progenitors to the developing heart tube. However, after looping, the mode of cardiac growth changes to expansion of committed cells, primarily through cardiomyocyte hyperplasia (Figure 1A and ref. 5).

In mice, cardiomyocyte proliferation peaks between embryonic day 10 (E10) and E12 and again at postnatal day 4 (P4) (6). Unlike proliferation that occurs during in utero development and the first few postnatal days, this second burst of cardiomyocyte cell-cycle entry occurs largely without cytokinesis, resulting in binucleate cardiomyocytes $(6,7)$. The frequency of mononuclear cardiomyocytes varies among mammalian species. Approximately $10 \%$ of rodent cardiomyocytes, but up to $78 \%$ of human cardiomyocytes, remain mononuclear $(8,9)$. However, even mononuclear human cardiomyocytes have elevated DNA content (3). At 1 year of age, $16 \%$ of human mononuclear cardiomyocytes are hyperdiploid, and this percentage increases to more than $50 \%$ after age 40 (10). The uncoupling of DNA synthesis from karyokinesis and cytokinesis is generally thought to correlate with a shift in cardiac growth from hyperplasia to primarily physiologic hypertrophy (Figure 1B). Recent reports describe a third burst of cardiomyocyte proliferation occurring in the subendocardial zone of the murine ventricular wall 14 days after birth (11). Proliferation of binucleated cardiomyocytes helps drive this growth, raising the possibility that hyperdiploid cardiomyocytes are capable of hyperplastic growth. The concept of significant new cardiomyocyte creation occurring in this preadolescent phase has been contested $(7,12,13)$.

Mechanistically, cessation of hyperplastic cardiomyocyte growth is thought to occur via an acquired cell-cycle arrest (Figure 2). Cyclins and cyclin-dependent kinases (CDKs) specific to G1/S and $\mathrm{G} 2 / \mathrm{M}$ phases are downregulated during the first week of life in mice. Meanwhile, G1 cyclins and CDKs are upregulated (14-16). The transcription factor MEIS1 likely plays an important role in upregulating cell-cycle inhibitors during cardiomyocyte maturation (Table 1 and ref. 17). MEIS1 binds the Ink4b-Arf-Ink4a and P21 loci in vivo and can activate their regulatory sequences. Remarkably, deleting Meis1 from adult mouse cardiomyocytes reportedly increases the 
A Cardiac development
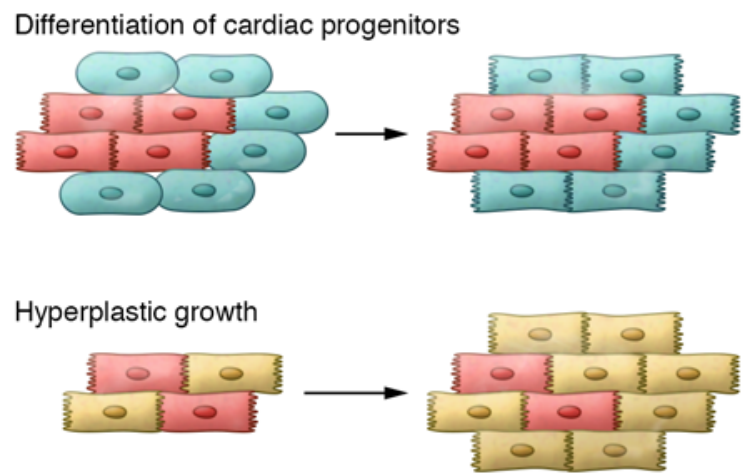

B Adult growth and maintenance

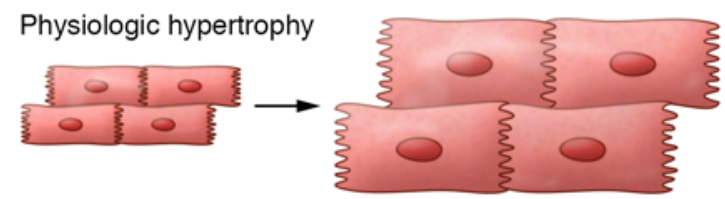

Pathologic hypertrophy

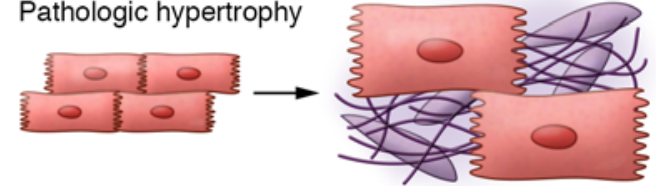

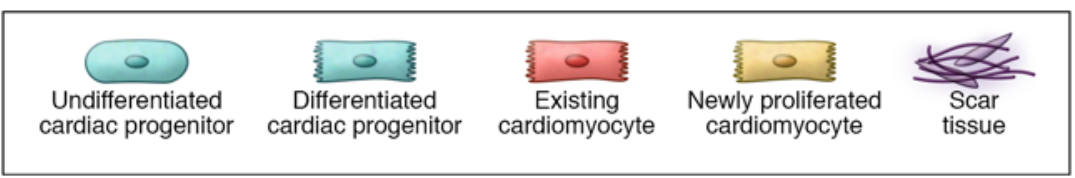

Figure 1. Modes of cardiomyocyte growth. (A) During cardiac development, cardiac growth occurs through addition of new cardiomyocytes via progenitor cell differentiation (top) followed by hyperplastic growth (bottom). Undifferentiated progenitor cells give rise to cardiac progenitors at the heart tube stage, followed by hyperplastic proliferation of cardiomyocytes during the remainder of development. (B) In contrast, the adult mammalian heart grows predominantly by either physiologic hypertrophy (top) or pathologic hypertrophy (bottom). With physiologic hypertrophy, the heart uniformly enlarges without organ fibrosis. Pathologic hypertrophy occurs after injury and is marked by fibrosis and heterogeneous cellular hypertrophy.

percentage of cycling cardiomyocytes (17). Other factors reported to contribute to acquired cell-cycle arrest are disassembly of centrosomes and methylation of developmental loci $(18,19)$.

The transition of cardiac growth from cardiomyocyte hyperplasia to hypertrophy may be part of the functional reprogramming of cardiomyocytes in response to increasing physiologic demands. Systolic blood pressure nearly doubles during the first week of life in mice, and a comparison across species suggests a relationship of greater cardiac workload to increasing cardiomyocyte DNA content. Adult rodents and humans have a 25 -fold higher blood pressure than adult zebrafish $(20,21)$. Perhaps accordingly, while approximately $90 \%$ of adult murine cardiomyocytes are binucleate and the majority of human cardiomyocytes are hyperdiploid, more than $95 \%$ of zebrafish cardiomyocytes are mononuclear and diploid $(6,22)$. Further, adult giraffes have nearly twice the blood pressure of mice and humans; giraffe cardiomyocytes also average 4 nuclei per cell and are likely to be at least octoploid (23). Metabolic shifts occur during the transition from a hypoxic fetal environment to the oxygen-rich postnatal environment. Corresponding with increases in mitochondrial density, greater levels of ROS may directly promote cell-cycle arrest (24). Finally, sarcomeres are remodeled to incorporate new adult protein isoforms. Alterations to cardiac compliance through mutations of sarcomeric proteins or extracellular matrix may also modulate the transition of cardiac growth from hyperplasia to hypertrophy $(25,26)$. Together, these data suggest a bias towards endoreplication and hypertrophic growth with increasing cardiomyocyte workload.

Intriguingly, recent work indicates that reducing cardiac workload can redirect cardiomyocyte growth towards hyperplasia. Histologic techniques suggest that significant increases in cardiomyocyte proliferation and cytokinesis occur after ventricular unloading in patients with heart failure supported by a left ventricular assist device (LVAD) $(27,28)$. These findings raise hope that LVAD therapy could be a platform for regenerative strategies in select patients.

\section{Homeostatic cardiac turnover}

Whereas cardiomyocyte renewal was once thought to be absent in the adult mammalian heart, recent studies suggest a low level of cardiomyocyte turnover. Historically, cardiomyocyte turnover had been measured by incorporation of ${ }^{3} \mathrm{H}$-thymidine or nucleoside analogs. These early studies suggested a low rate of cardiomyocyte replacement, but definitive conclusions were limited by the imprecision of histological techniques for identifying cardiomyocyte nuclei $(7,29)$. Subsequent strategies to estimate the rate of cardiomyocyte cycling have focused on colocalization of cardiomyocyte markers with indicators of proliferation. Soonpaa et al. combined a transgenic nuclear mark for cardiomyocytes with ${ }^{3} \mathrm{H}$-thymidine labeling of cells undergoing DNA synthesis and reported cycling of $0.0005 \%$ of cardiomyocytes in the uninjured adult mouse heart (30). Other groups have developed orthogonal techniques to identify cycling cardiomyocytes. Senyo et al. pulsed mice with ${ }^{15} \mathrm{~N}$-thymidine and used mass spectrometry to identify cells with increased ${ }^{15} \mathrm{~N}$ content in their nucleus as evidence for DNA synthesis. Coupling this approach with highmagnification microscopy enabled identification of cycling cardiomyocytes in the mouse heart. They reported a rate of cardiomyocyte renewal of $0.76 \%$ per year (31). More recently, Kimura et al. have developed a genetic fate mapping approach to follow hypoxic cardiomyocytes over 1 month as a surrogate for myocyte cycling. This work estimated a cardiomyocyte renewal rate of approximately $1 \%$ annually (32). 


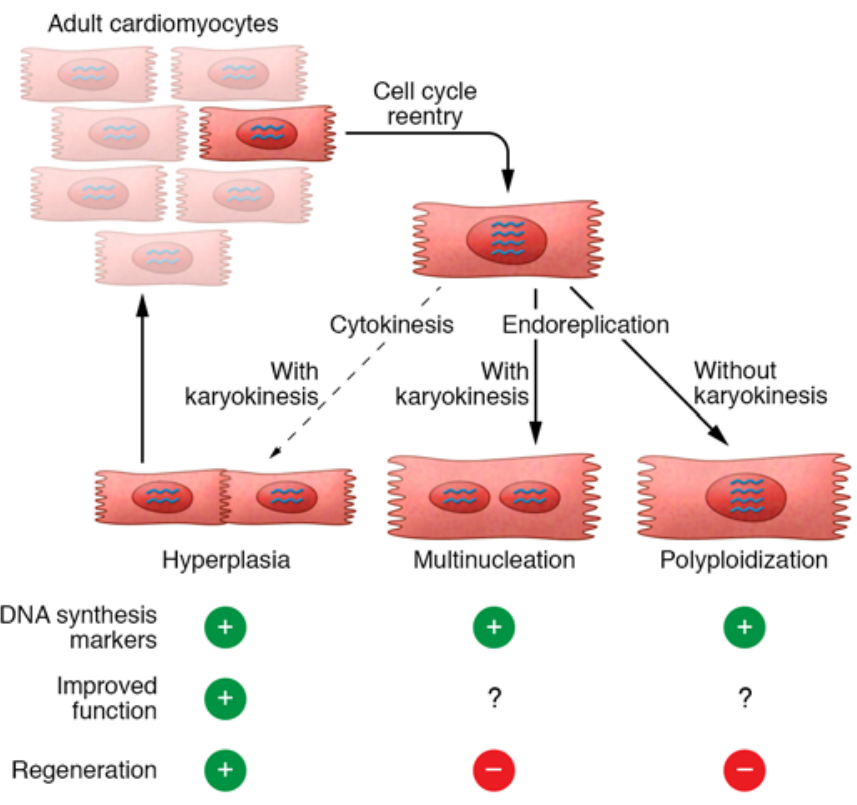

Figure 2. Postnatal cardiomyocyte cycling in the adult mammalian heart and challenges in assessing cardiac regeneration. In the adult mammalian heart, the majority of cardiomyocytes are quiescent and do not cycle. Rare cardiomyocytes that do cycle preferentially undergo endoreplication rather than complete mitosis. This creates challenges in assessing mammalian heart regeneration. Indices of cardiomyocyte cycling can mark true regeneration through hyperplastic growth but are more likely to mark cardiomyocytes that undergo endoreplication without making new muscle. Similarly, increased ventricular function is associated with regeneration following the creation of new muscle, but may be increased following endoreplication as well.

The basal rates of cardiomyocyte turnover estimated in rodent hearts are consistent with retrospective analyses of human tissue. Bergmann et al. used postmortem tissue samples and took advantage of atmospheric ${ }^{14} \mathrm{C}$ levels to assign birth dates for cardiomyocyte nuclei. By comparing the age of DNA in cardiomyocyte nuclei with the age of the host, they estimate an annual rate of cardiomyocyte cycling of approximately $0.3 \%$ to $1 \%$ per year ( 9 , 33). Although this rate seems low, homeostatic turnover ostensibly maintains a constant total cardiomyocyte number and contributes up to $39 \%$ of all cardiomyocytes in a 75 -year-old person (9). Similarly, Mollova et al. employed a histological tour de force with tissue obtained from unused transplant donor hearts to estimate the rate of cardiomyocyte cycling in young humans at $1.9 \%$ per year (10). Based on the striking concordance of experimental results across species, it is now accepted that the adult mammalian heart retains a limited number of differentiated cardiomyocytes capable of reentering the cell cycle, and cardiomyocyte renewal occurs at a rate of approximately $1 \%$ annually under homeostatic conditions.

\section{Growth responses following injury}

After injury, both mammalian and zebrafish hearts activate a cardiac growth response. However, the outcomes following cardiac injury are very different. Zebrafish regenerate lost cardiac tissue following mechanical amputation, genetic ablation of cardiomyocytes, or cryoinjury to the surface of the heart (34-38). In contrast, very little, if any, regeneration occurs in the adult mammalian heart with injuries such as myocardial infarction (MI). Instead, the mammalian heart is unable to replenish lost cardiomyocytes and undergoes replacement fibrosis. Over time, the mammalian heart typically progresses to ventricular dysfunction and heart failure (Figure 1B).

Although the adult mouse heart has a limited regenerative capacity, embryonic and neonatal mice are capable of recovering lost cardiomyocytes through direct cardiomyocyte proliferation that is analogous to zebrafish. Genetic ablation of fetal cardiomyocytes results in replacement of lost cardiomyocytes through compensatory increases in cardiomyocyte proliferation $(39,40)$. Regenerative capacity of the mammalian heart extends into the early neonatal period. Within the first week of life, the mouse heart is capable of regeneration following ventricular resection, $\mathrm{MI}$, or genetic ablation of cardiomyocytes (41-43). Interestingly, regeneration does not seem to occur following severe injuries such as cryoablation (44). Similar to zebrafish, regeneration in neonatal mice occurs by cardiomyocyte proliferation, sarcomere disassembly, and epicardial activation $(41,45,46)$. Thus, the basic regenerative program is likely conserved from fish to mammals. Interestingly, 7-day-old mice are unable to effect heart regeneration (41). If the basic regenerative machinery exists, why can't the adult mouse heart meaningfully regenerate? Ultimately, understanding factors that impact fate decisions of the major cardiac cell types following injury may provide insights to facilitate mammalian heart regeneration.

Cardiomyocyte responses to injury. The classic response of the mammalian cardiomyocyte to injury is hypertrophy. According to LaPlace's law, ventricular wall stress can be held constant by compensatory thickening of the cardiac wall. This hypertrophy can be either physiologic or pathologic. Physiologic hypertrophy occurs with exercise or pregnancy and is morphologically characterized by uniform organ enlargement with proportional increases in chamber volume (Figure 1B). Of note, physiologic hypertrophy occurring with exercise likely involves a combination of hypertrophic and rare hyperplastic growth $(47,48)$. In contrast, pathologic hypertrophy occurs in response to a variety of stimuli such as mechanical load or neuroendocrine factors and is maladaptive over time (Figure 1B and ref. 49). It is uncertain whether stimuli associated with physiologic hypertrophy lead to increased hyperplastic cardiomyocyte growth compared with stimuli that lead to pathologic hypertrophy.

The molecular hallmark of pathologic hypertrophy in mammals is induction of a fetal gene expression program. Classically, this involves switching from an adult $\alpha$-myosin heavy chain $(\alpha-\mathrm{MHC})$ to a fetal $\beta$-MHC isoform $(49,50)$. Induction of a fetal gene program may be protective under acute stress, but prolonged activation may be deleterious (51). For example, genetic reduction of the developmental transcription factor GATA4 using dominantnegative or knockout approaches in mice results in deterioration of cardiac function with pressure overload (52-55). In contrast, much therapeutic effort has gone toward reducing markers of the fetal gene expression program, such as B-type natriuretic peptide (56).

Following injury, zebrafish cardiomyocytes also reexpress a variety of developmental factors such as natriuretic peptides, embryonic MHCs, and signaling factors (57-61). However, zebrafish cardiomyocytes proliferate following injury and ultimately give rise to new cardiomyocytes $(45,46)$. Genetic lineage tracing 
Table 1. Pathways to control mammalian cardiomyocyte proliferation in different developmental contexts

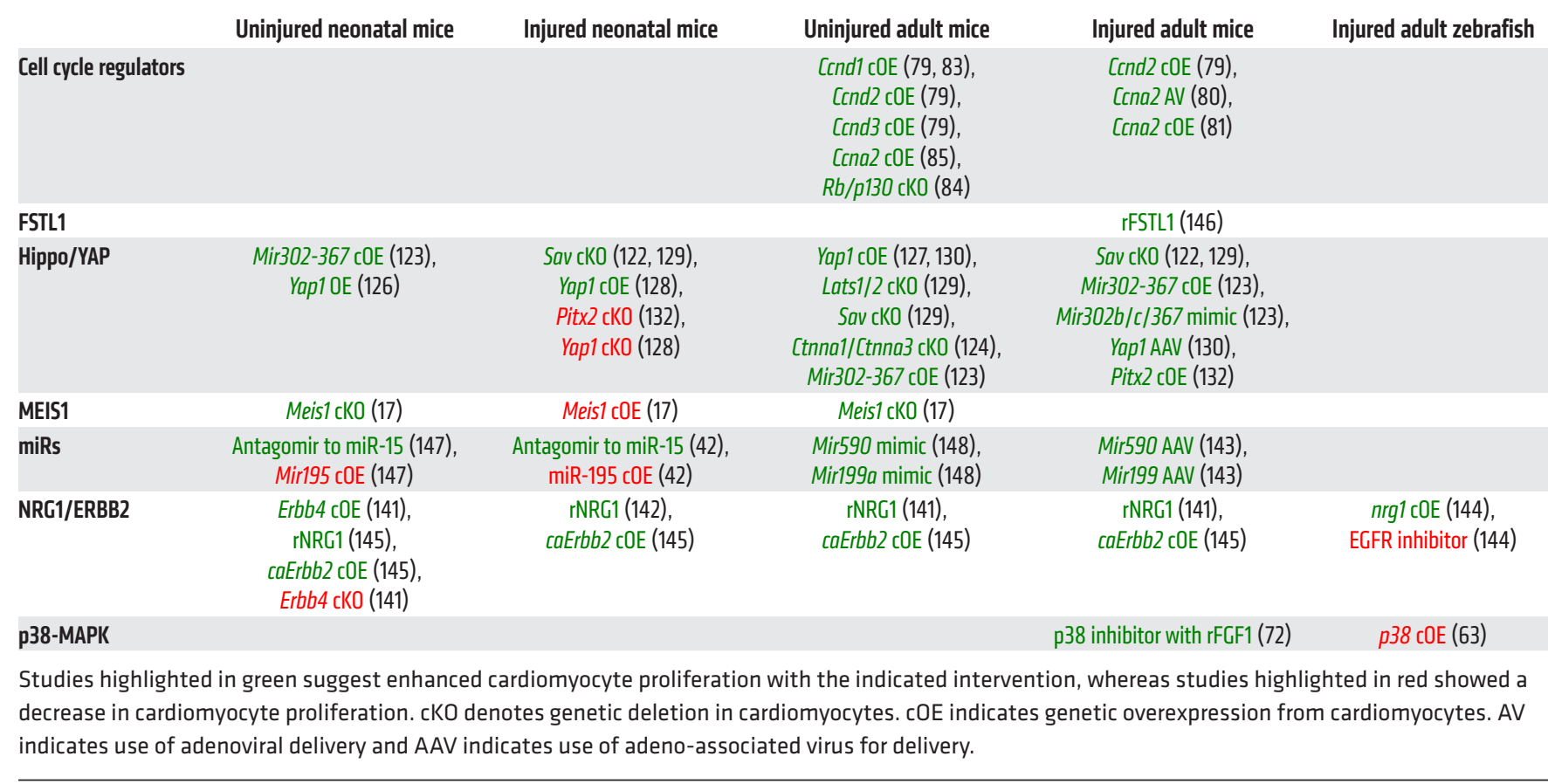

experiments demonstrate that cardiomyocytes which reexpress gata4 primarily contribute to new muscle during regeneration $(45,58)$. Induction of a cardiac developmental program that includes Gata4 appears to be a key component of the regenerative program in zebrafish. For example, loss of Gata4 function during regeneration reduces cardiomyocyte proliferation and blocks regeneration (58).

Despite differing modes of growth following injury, cardiomyocytes in zebrafish and mammalian hearts activate many similar pathways. Inhibition of Stat 3 signaling, inhibition of NF- $\kappa \mathrm{B}$ signaling, or activation of p38 signaling all reduce cardiomyocyte proliferation and impair regeneration in zebrafish (62-64). In mammalian systems, STAT3 has been shown to be required for hypertrophy of cardiomyocytes, and genetic attenuation of STAT3 signaling results in rapid progression to heart failure following cardiac stress (65-69). Similarly, NF- $\mathrm{BB}$ signaling contributes to the reexpression of developmental factors and is considered to be essential to pathologic hypertrophy (70). Likewise, p38 inhibition following experimental MI in mice promotes hypertrophy $(63,71-73)$.

If the same molecular pathways are induced, why do zebrafish cardiomyocytes proliferate while adult mammalian cardiomyocytes hypertrophy? A likely possibility is decreased competence of mammalian cardiomyocytes to complete the cell cycle (Figure 2). Genes promoting cell-cycle reentry, such as $M y c, C c n d 1$, and $C d k 2$, are upregulated during pathologic hypertrophy $(74,75)$. Similarly, genes that suppress cycling, such as $P 21$ and $P 27$, are downregulated (76). Despite these molecular changes, cardiomyocytes rarely complete mitosis $(29,31)$. In the aforementioned study by Senyo et al. using a ${ }^{15} \mathrm{~N}$ pulse to label cycling cardiomyocytes, up to $23 \%$ of murine cardiomyocytes cycle in the border zone after experimental infarction. Interestingly, only $3.2 \%$ of cycling cardiomyocytes remain diploid following infarction, suggesting that endoreplication occurs in the majority of cycling cardiomyocytes (31). Highresolution analysis in transgenic mice expressing the scaffolding protein anillin fused to EGFP also confirms a bias of cycling myocytes towards increased ploidy compared with hyperplasia following MI (77). Perhaps the strongest data for diminished competence for mitosis come from studies overexpressing oncogenes in cardiomyocytes. Forced expression of Ccnd2 or Ccna2, or genetic depletion of $R b$ and $p 130$, increases the number of cardiomyocytes undergoing DNA synthesis, but the overall number of cycling cardiomyocytes is modest (refs. 78-85 and Table 1). Even more striking, transgenic overexpression of $M y c$ in cardiomyocytes during embryogenesis results in cardiomegaly by hyperplasia, but overexpression of $M y c$ in adult murine cardiomyocytes results in cardiomegaly by hypertrophy $(86,87)$. Together, these data suggest that myocyte competence for cell-cycle completion is a limiting factor for mammalian heart regeneration.

Epicardial responses to injury. The epicardium is a mesothelial sheet that covers the heart. Lineage tracing experiments during development indicate that the epicardium gives rise to mural cells of the coronary vasculature, to cardiac fibroblasts, and to pericardial fat (88-92). Earlier reports using $W t 1$ or $T b x 18$ as epicardial markers suggested that the epicardium could transdifferentiate into cardiomyocytes, but use of other epicardial markers, such as $T c f 21$, have not confirmed these results (93-95). In addition to its cellular contributions, the epicardium also has a major role during development as a source for paracrine factors. Epicardial sources of retinoic acid and FGFs, for instance, stimulate cardiomyocyte proliferation and coronary vasculogenesis $(96,97)$.

Following injury, the epicardium appears to have roles similar to those during cardiac development. After experimental 
MI in mammals, epicardial cells appear to give rise primarily to scar-forming fibroblasts and to peri-infarct adipocytes (89, 92, 98-100). In zebrafish, however, epicardial cells proliferate and give rise to new epicardial cells, mural cells that support nascent vasculature, and myofibroblasts $(95,101,102)$. Genetic ablation studies of $t c f 21^{+}$epicardial and epicardium-derived cells in zebrafish have demonstrated the indispensable role for the epicardium in heart regeneration. Genetic ablation of the epicardium not only prevents regeneration but also reduces cardiomyocyte proliferation, suggesting an important paracrine role for $t c f 21^{+}$cells to support a regenerative program (103). Similarly, conditioned media from epicardial cells may promote myocardial recovery in mice (98). Comparative work on responses of the epicardium to injury in mice and zebrafish has indicated more dramatic upregulation of raldh2, the key enzyme for retinoic acid synthesis, in the zebrafish epicardium compared with mice. Thus, the mammalian epicardium may not support a regenerative program as efficiently as in zebrafish (104). Future work to identify factors that influence epicardial cell fates and factors involved in the myocardial-epicardial dialogue may prove vital for biasing cardiac growth towards regeneration in the mammalian heart.

Vascular responses to injury. Following injuries such as $\mathrm{MI}$ to the mammalian heart, tissue hypoxia results in HIF1A activation and induction of vasculogenic factors, including VEGF and angiopoietin. However, while small collateral vessels can form with chronic ischemia, the vasculogenic response in the mammalian heart is generally limited. Zebrafish, by contrast, display a marked vasculogenic reaction just a few hours after injury (105). Following injury, new vascular endothelium emerges from preexisting endothelium (106). Mechanistically, FGF, Notch, and VEGF signaling contribute to vasculogenesis following ventricular injury in zebrafish (57, $105,106)$. Recent work has also established the importance of the chemokine $c x c l 12 b$ and its receptor $c x c r 4 a$ to vasculogenesis during cardiac growth and regeneration (107). Defects in FGF, Notch, VEGF, or chemokine signaling pathways result in impaired vasculogenesis and defective zebrafish heart regeneration (57, 105-107). Importantly, inhibition of Notch or VEGF pathways also results in decreased cardiomyocyte proliferation, illustrating the importance of vasculogenesis in tuning heart regeneration $(105,106)$

Inflammatory responses to injury. Among the earliest response to myocardial injury is the inflammatory response. Macrophages, in particular, have been identified as key mediators of the inflammatory response with important contributions to ventricular remodeling following cardiac injury (108). Not surprisingly, macrophages likely modulate regenerative responses as well. Macrophages are recruited to the injury during zebrafish heart regeneration (35). Although it is unclear whether macrophage recruitment is required for zebrafish heart regeneration, the macrophage response appears to be critical for regeneration in other tissues, such as fins $(109,110)$. Similar to results from other regenerating tissues, pharmacologic ablation of granulocytes blocks regeneration in the neonatal mouse following apical resection or by genetic ablation of cardiomyocytes. These effects appear to be mediated, in part, by embryo-derived macrophages, which may work by stimulating an angiogenic response $(43,111)$.

Contributions of nerves. Nerves have long been known to guide salamander limb regeneration (112). Recent work explored the importance of cardiac innervation to heart regeneration. In zebrafish, reducing innervation by overexpressing the neurorepellent sema3aa or pharmacologic inhibition of cholinergic signaling impairs heart regeneration by decreasing cardiomyocyte proliferation (113). Further, parasympathetic denervation or sympathectomy compromises regeneration in neonatal rodents (113, 114). The mechanisms by which nerves modulate cardiac regenerative responses await discovery. However, nerves are likely to either directly or indirectly affect the growth factor milieu and inflammatory response following injury (113).

Stem cell contributions to cardiac growth following injury. Over the past 30 years, much work has focused on identifying a progenitor cell population in the adult mammalian heart. Several stem cell populations have been proposed, including $\mathrm{Kit}^{+}, \mathrm{Sca}^{+}, \mathrm{PDGFRA}^{+}$, or Isl1 ${ }^{+}$cells (115). Initial studies using genetic lineage tracing to mark preexisting cardiomyocytes showed a gradual dilution of preexisting cardiomyocytes with new cardiomyocytes, suggesting that a progenitor cell population contributes to de novo cardiomyocytes during homeostasis and following myocardial injury (116). However, refinement of this genetic dilution approach to focus on cycling cells revealed that most newly formed cardiomyocytes are derived from preexisting cardiomyocytes (31). Technically, these results do not rule out transdifferentiation, without proliferation, of a resident progenitor cell population into cardiomyocytes. However, when taken together with the results of lineage tracing studies for putative stem cell populations, a significant contribution to de novo cardiomyocytes by a progenitor pool is unlikely. For instance, exhaustive lineage tracing of $\mathrm{Kit}^{+}$cells by 3 separate groups shows a negligible contribution of these cells to cardiomyocytes following injury in the adult mouse heart (117-119). In contrast, progenitor cell populations may contribute to other cardiac tissue types, such as coronary endothelium $(117,120)$.

\section{Adult mammalian heart regeneration}

Recent seminal discoveries in developmental and stem cell biology have now made therapeutic heart regeneration a possibility (121). Recognition of cardiomyocyte proliferative capacity has created fervor to identify cardiac mitogenic influences capable of augmenting endogenous regenerative potential. Two emerging themes are the relevance of developmental growth pathways to effect heart regeneration and the tendency for factors that promote regeneration in zebrafish and neonatal mice to also promote hypertrophy in the adult mammalian heart. Here, we review factors that may enhance endogenous regenerative responses.

Hippo signaling. The Hippo pathway was identified during a screen for regulators of organ size in Drosophila. In general, the transcription factors YAP and TAZ promote organ growth and are inhibited by Hippo kinase activity (Table 1). YAP1 signaling is also responsive to alterations in the cytoskeleton, and altering cellular structure may be an alternative way to modulate YAP1 activity (122124). Heallen et al. originally described the critical role for Hippo signaling in determining cardiac size and cardiomyocyte numbers during development (125). Excess YAP activity by deletion of Hippo kinases Mst1/2 or Lats2, deletion of the adaptor protein Sav1, or overexpression of Yap1 in cardiomyocytes leads to cardiomyocyte hyperplasia and cardiomegaly in fetal mice (125-127). Conversely, deletion of Yap1 in cardiomyocytes during development leads to 
hypoplastic hearts with impaired cardiomyocyte proliferation (126, 127). The decrease in YAP activity in the postnatal mammalian heart suggests that Hippo signaling may contribute to cardiomyocyte cell-cycle arrest in the adult mammalian heart. Indeed, excess YAP activity extends the window for cardiomyocyte proliferation in the neonatal mouse heart and enhances cardiomyocyte proliferation in the adult mouse heart $(126,128-130)$. Mechanistically, postnatal YAP activity is likely regulated by decreased acetylation of the transcription cofactor VGLL4, leading to degradation of the YAP1 binding partner TEAD1 (131). Modulation of the Hippo pathway seems to affect regenerative responses. Deletion of Yap1 from fetal cardiomyocytes impairs regeneration of the neonatal mouse heart following apical resection or MI. Conversely, expression of a constitutively active form of Yap1 or deletion of Sav1 leads to evidence of enhanced regeneration following cardiac injury in 1-week-old mice (128). Further, infarct size is reduced by nearly 25\% following left anterior descending artery ligation in Sav1deficient adult mouse hearts, and this is associated with enhanced cardiomyocyte cycling (129). Similarly, overexpression of activated YAP at the time of infarction in mice improves survival and ventricular function (130). Mechanistically, YAP likely interacts with the developmental factor PITX2 to induce genes that scavenge ROS. Accordingly, overexpression of Pitx 2 can improve ventricular recovery following cardiac injury in neonatal and adult mice (132). Although increased YAP activity likely favors hyperplastic growth under homeostatic conditions in the adult mammalian heart, this bias towards cardiomyocyte hyperplasia over hypertrophy has not been demonstrated during pathologic states, such as after MI in adult mice $(126,129,130)$. Other studies have associated excess YAP activity with hypertrophy (133-135). Nonetheless, modulation of the Hippo-YAP axis is a promising approach for achieving adult mammalian heart regeneration.

Neuregulin signaling. A second developmental pathway that has recently gained traction to effect heart regeneration is the neuregulin 1/ERBB2/ERBB4 (NRG1/ERBB2/ERBB4) pathway (Table 1). Glial growth factors were first identified as mitogens from pituitary extracts and were later found to be ligands for the ERBB family of receptors $(136,137)$. Genetic deletion studies have confirmed the requirement for the NRG1/ERBB axis during heart development. Mice deficient for Nrg1 or its coreceptors Erbb2 and Erbb4 have defects in cardiomyocyte proliferation that result in hypoplastic hearts (138-140). Bersell and colleagues identified NRG1 in a screen for growth factors that could stimulate adult cardiomyocyte proliferation in vitro. Following MI, they showed that recombinant NRG1 could stimulate postnatal cardiomyocyte cycling by more than 4.4 -fold and improve ventricular function. Interestingly, Bersell and colleagues noted that mononuclear cardiomyocytes are capable of cycling much more efficiently than binucleate cardiomyocytes (141). Consistent with this notion, recombinant NRG1 more potently induces cardiomyocyte cycling in the neonatal mouse prior to binucleation (142).

In zebrafish, $n r g 1$ is induced during heart regeneration in perivascular cells. By contrast, the cellular source in mice is thought to be the endocardium (143). Transgenic overexpression of $n r g 1$ in zebrafish is sufficient to induce massive cardiac hyperplasia (144). Similarly, expression of a constitutively active Erbb2 (caErbb2) can induce cardiomyocyte cycling in the neonatal, juvenile, and adult mouse heart. While cardiomyocyte cycling is more efficient in mononucleated cardiomyocytes, D'Uva and colleagues found that binucleate cardiomyocytes could also enter the cell cycle. Interestingly, increased cycling is also associated with increased hypertrophy. Nonetheless, transient induction of caErbb2 can enhance cardiomyocyte cycling by more than 4 -fold and can increase ventricular function after MI (145).

Epicardial FSTL1. As mentioned earlier, the epicardium is regarded as a source for paracrine factors that support organ growth during development. Using a coculture system of epicardial cells with embryonic stem cell-derived cardiomyocytes, Wei et al. found that the epicardial secretome can boost cardiomyocyte proliferation in vitro. When added to an engineered patch that is applied to infarcted mouse hearts, the epicardial secretome improves ejection fraction. Interestingly, chamber dimensions are not substantially improved by addition of the epicardial secretome compared with the patch alone. To identify which factors in the epicardial secretome confer beneficial effects, Wei et al. performed mass spectrometry and identified FSTL1 as a putative cardiomyocyte mitogen (Table 1). Administration of recombinant FSTL1 via the cardiac patch recapitulates the effects of the epicardial secretome and improves ventricular function following MI in mice and swine (Table 1). While recombinant FSTL1 nearly doubles cardiomyocyte cycling, it seems to promote hypertrophy when overexpressed in myocytes, which are the most abundant source of endogenous FSTL1. Differential effects of myocardial and epicardial FSTL1 may be related to posttranslational processing (146).

Developmental miRs. By comparing miR profiles of $\mathrm{P} 1$ and $\mathrm{P} 10$ hearts, Porrello et al. identified a cluster of miR-15 family members induced during acquired cardiomyocyte quiescence. Of these members, miR-195 is the most dynamic, being upregulated 6-fold during the first week of life in rodents. Transgenic overexpression of miR-195 in cardiomyocytes during development leads to cardiac hypoplasia (147). Similarly, using an antagomir directed against miR-15 family members can promote cardiomyocyte cycling in the neonatal heart and improve cardiac function after experimental infarction with a greater than 4 -fold increase in cardiomyocyte cycling (Table 1 and ref. 42).

The miR cluster Mir302/367 is also dynamic during development, but expression levels peak at E9.5 and decrease during maturation. While loss of the Mir302/367 locus during development suppresses cardiomyocyte cycling, overexpression enhances cycling and increased numbers of mononuclear and multinucleate cardiomyocytes. Remarkably, transgenic overexpression of Mir302/367 resulted in a greater than 60-fold increase in $\mathrm{H}^{3} \mathrm{P}^{+}$cardiomyocytes (Table 1). However, rather than improving function, animals overexpressing Mir302/367 develop progressive cardiac dysfunction with apparent defects in cardiomyocyte maturation. By contrast, intermittent overexpression via administration of a miR-302 mimic promotes ventricular recovery following infarction without progressive chamber dilation. Mechanistically, miR-302 appears to directly regulate cell-cycle genes as well as inactivate Hippo signaling by repressing MST1/2 and LATS kinases (123).

Other groups have screened chemical libraries for proregenerative miRs. Using such an approach, Eulalio et al. demonstrated that miR-199 and miR-590 stimulate neonatal and adult cardiomyocyte proliferation in vitro. Following infarction, these miRs 
can stimulate DNA synthesis in vivo and improve cardiac function (Table 1 and ref. 148). The roles of miR-199 and miR-590 during cardiac development have yet to be described.

Hypoxic conditioning. Extending the concept that the hypoxic fetal circulation may promote cardiomyocyte competence for hyperplastic growth, recent work suggests that hypoxic conditioning may be an approach to nudge the regenerative capacity of the adult mammalian heart (149). Nakada et al. found that exposing mice to $7 \%$ oxygen for 3 weeks after coronary ligation decreased scar size and improved left ventricular function. Hypoxic conditioning was associated with evidence of increased cycling by cardiomyocytes $(\sim 1.3 \%$ in the hypoxic group versus $0 \%$ in the normoxic group). The idea that tissue hypoxia might stimulate meaningful heart regeneration is perplexing, as ischemic heart disease is the most common cause of ventricular dysfunction worldwide and coronary revascularization extends survival in patients with ischemic cardiomyopathy (150).

\section{Technical limitations to assessing mammalian heart regeneration}

Although much of the above work is promising, important caveats must be considered when assessing regeneration of the mammalian heart. The strict definition of regeneration is the replacement of lost or damaged tissue with new tissue. To date, there are no reliable techniques to differentiate newly regenerated cardiac muscle from preexisting muscle in the mammalian heart. Unlike zebrafish, in which direct removal of muscle leads to replacement with new muscle via a local response, mammalian models of regeneration undergoing rapid animal and cardiac growth might be recovering muscle primarily or exclusively away from the injury $(151,152)$. Therefore, distinguishing changes in muscle content can be confounded by the type and extent of injury. In the absence of direct methods to identify new muscle, most regenerative studies rely on indirect surrogates for regeneration, such as quantification of rare cardiomyocytes that reenter the cell cycle. Assays for cardiomyocytes that incorporate BrdU, that express proliferative marks such as H3P or PCNA, that express cytokinesis markers such as AURKB or MKLP1, or that incorporate heavy isotopes of nitrogen in pulse-chase experiments have been used to assess heart regeneration $(10,17,31,141,153)$. While much prior work relied on colocalization of proliferative marks with sarcomeric proteins, precisely estimating rates of cardiomyocyte cycling is difficult without the use of cardiomyocyte-specific nuclear markers $(9,29)$. This issue is compounded by the rarity of proliferating cardiomyocytes such that even 1 or 2 incorrectly classified nuclei lead to large errors in relative rates of cardiomyocyte cycling. For instance, an intervention that increases the number of cycling cardiomyocytes from a single myocyte per low-powered field to 3 myocytes per field could be reported as increasing cardiomyocyte proliferation by $200 \%$. However, the absolute contribution of the intervention to regeneration is much more modest.
Several groups have recently developed transgenic mouse strains to precisely identify cardiomyocyte nuclei in the mammalian heart, which should improve the accuracy for determining rates of cardiomyocyte proliferation. However, even with better methods to identify cycling cardiomyocytes, these assays only suggest newly formed cardiac muscle (Figure 2 and refs. 7, 154). Markers of cell-cycle reentry are unable to distinguish hyperplastic growth from cycling cells that do not complete karyokinesis or cytokinesis. For adult mammalian cardiomyocytes, the differentiation of hyperplastic growth from endoreplication is of critical importance, as the majority of cycling cardiomyocytes do not result in new cardiomyocytes. In addition to proliferative marks, others have used ventricular function to assess regeneration. However, ventricular function is often dependent on multiple factors not necessarily related to regeneration, such as loading conditions. Similarly, molecules that affect the progression of compensatory hypertrophy and cardiac remodeling can also result in improved function. Lineage tracing approaches using multicolor clonal analysis or mosaic analysis with double markers have the potential to clearly resolve hyperplastic growth, but the rarity of cardiomyocyte cycling in the adult heart limits the sensitivity of approaches that rely on mosaic labeling (155). As more work is done to potentiate mammalian heart regeneration, more sensitive tools to assess bona fide regeneration will be needed.

\section{Summary}

Achieving therapeutic heart regeneration is a critical biomedical goal with wide societal impact. While striving for these lofty goals, a better understanding of the mechanisms that regulate cardiac growth and endogenous heart regeneration will only smooth the way. Future work to identify additional factors that promote regenerative responses and methods for delivery of these factors may hold the key to making regeneration more efficient.

\section{Acknowledgments}

We thank Amy Dickson for assistance with figures. We thank Poss lab members for comments on the manuscript. We apologize to our colleagues whose work we could not discuss owing to space limitations. RK is supported by an NIH Mentored Clinical Scientist Award, K08-HL116485, the Walker P. Inman Endowment, and the Edna and Fred L. Mandel, Jr. Foundation. KDP acknowledges support by grants from the NIH (R01-HL081674, R01-HL136182, and R01-HL131319), Fondation Leducq, and the American Heart Association.

Address correspondence to: Ravi Karra, Box 3126, Duke University Medical Center, Durham, North Carolina 27710, USA. Phone: 617.997.8326; E-mail: ravi.karra@duke.edu. Or to: Kenneth D. Poss, Department of Cell Biology, Duke University Medical Center, Durham, North Carolina 27710, USA. Phone: 919.681.8457; E-mail:kenneth.poss@duke.edu.
1. Braunwald $\mathrm{E}$. The war against heart failure: the Lancet lecture. Lancet. 2015;385(9970):812-824.

2. Braunwald E. Heart failure. JACC Heart Fail.

2013;1(1):1-20.

3. Liu J, Stainier DY. Zebrafish in the study of early car- diac development. Circ Res. 2012;110(6):870-874.

4. de Boer BA, van den Berg G, de Boer PA, Moorman AF, Ruijter JM. Growth of the developing mouse heart: an interactive qualitative and quantitative 3D atlas. Dev Biol. 2012;368(2):203-213.
5. Meilhac SM, Kelly RG, Rocancourt D, EloyTrinquet S, Nicolas JF, Buckingham ME. A retrospective clonal analysis of the myocardium reveals two phases of clonal growth in the developing mouse heart. Development. 
2003;130(16):3877-3889.

6. Soonpaa MH, Kim KK, Pajak L, Franklin M, Field LJ. Cardiomyocyte DNA synthesis and binucleation during murine development. Am J Physiol. 1996;271(5 pt 2):H2183-H2189.

7. Hirai M, Chen J, Evans SM. Tissue-specific cell cycle indicator reveals unexpected findings for cardiac myocyte proliferation. Circ Res. 2016;118(1):20-28.

8. Li F, Wang X, Capasso JM, Gerdes AM. Rapid transition of cardiac myocytes from hyperplasia to hypertrophy during postnatal development. J Mol Cell Cardiol. 1996;28(8):1737-1746.

9. Bergmann O, et al. Dynamics of cell generation and turnover in the human heart. Cell. 2015;161(7):1566-1575.

10. Mollova M, et al. Cardiomyocyte proliferation contributes to heart growth in young humans. Proc Natl Acad Sci U S A. 2013;110(4):1446-1451.

11. Naqvi $\mathrm{N}$, et al. A proliferative burst during preadolescence establishes the final cardiomyocyte number. Cell. 2014;157(4):795-807.

12. Soonpaa MH, Zebrowski DC, Platt C, Rosenzweig A, Engel FB, Field LJ. Cardiomyocyte cell-cycle activity during preadolescence. Cell. 2015;163(4):781-782.

13. Alkass K, Panula J, Westman M, Wu TD, GuerquinKern JL, Bergmann O. No evidence for cardiomyocyte number expansion in preadolescent mice. Cell. 2015;163(4):1026-1036.

14. Poolman RA, Brooks G. Expressions and activities of cell cycle regulatory molecules during the transition from myocyte hyperplasia to hypertrophy. J Mol Cell Cardiol. 1998;30(10):2121-2135.

15. Liu Z, Yue S, Chen X, Kubin T, Braun T. Regulation of cardiomyocyte polyploidy and multinucleation by CyclinG1. Circ Res. 2010;106(9):1498-1506.

16. Poolman RA, Gilchrist R, Brooks G. Cell cycle profiles and expressions of p21CIP1 AND P27KIP1 during myocyte development. Int J Cardiol. 1998;67(2):133-142.

17. Mahmoud AI, et al. Meis1 regulates postnatal cardiomyocyte cell cycle arrest. Nature. 2013;497(7448):249-253.

18. Zebrowski DC, et al. Developmental alterations in centrosome integrity contribute to the postmitotic state of mammalian cardiomyocytes. Elife. 2015;4:e05563.

19. Sim CB, et al. Dynamic changes in the cardiac methylome during postnatal development. FASEB J. 2015;29(4):1329-1343.

20. Le VP, Kovacs A, Wagenseil JE. Measuring left ventricular pressure in late embryonic and neonatal mice. JVis Exp. 2012;(60):3756.

21. Hu N, Yost HJ, Clark EB. Cardiac morphology and blood pressure in the adult zebrafish. Anat Rec. 2001;264(1):1-12.

22. Wills AA, Holdway JE, Major RJ, Poss KD. Regulated addition of new myocardial and epicardial cells fosters homeostatic cardiac growth and maintenance in adult zebrafish. Development. 2008;135(1):183-192.

23. Østergaard KH, et al. Left ventricular morphology of the giraffe heart examined by stereological methods. Anat Rec (Hoboken). 2013;296(4):611-621.

24. Puente BN, et al. The oxygen-rich postnatal environment induces cardiomyocyte cell-cycle arrest through DNA damage response. Cell. 2014;157(3):565-579.

25. Jiang J, et al. Cardiac myosin binding protein $C$ regulates postnatal myocyte cytokinesis. Proc Natl Acad Sci U S A. 2015;112(29):9046-9051.

26. Yahalom-Ronen Y, Rajchman D, Sarig R, Geiger $\mathrm{B}$, Tzahor E. Reduced matrix rigidity promotes neonatal cardiomyocyte dedifferentiation, proliferation and clonal expansion. Elife. 2015; $4: \mathrm{e} 07455$.

27. Wohlschlaeger J, et al. Hemodynamic support by left ventricular assist devices reduces cardiomyocyte DNA content in the failing human heart. Circulation. 2010;121(8):989-996.

28. Canseco DC, et al. Human ventricular unloading induces cardiomyocyte proliferation. J Am Coll Cardiol. 2015;65(9):892-900.

29. Soonpaa MH, Field LJ. Survey of studies examining mammalian cardiomyocyte DNA synthesis. Circ Res. 1998;83(1):15-26.

30. Soonpaa MH, Field LJ. Assessment of cardiomyocyte DNA synthesis in normal and injured adult mouse hearts. Am J Physiol.1997; 272(1 pt 2):H22O-H226.

31. Senyo SE, et al. Mammalian heart renewal by pre-existing cardiomyocytes. Nature. 2013;493(7432):433-436.

32. Kimura $\mathrm{W}$, et al. Hypoxia fate mapping identifies cycling cardiomyocytes in the adult heart. Nature. 2015;523(7559):226-230.

33. Bergmann O, et al. Evidence for cardiomyocyte renewal in humans. Science. 2009;324(5923):98-102.

34. Poss KD, Wilson LG, Keating MT. Heart regeneration in zebrafish. Science. 2002;298(5601):2188-2190.

35 . Wang J, et al. The regenerative capacity of zebrafish reverses cardiac failure caused by genetic cardiomyocyte depletion. Development. 2011;138(16):3421-3430.

36. González-Rosa JM, Martín V, Peralta M, Torres M, Mercader N. Extensive scar formation and regression during heart regeneration after cryoinjury in zebrafish. Development. 2011;138(9):1663-1674.

37. Chablais F, Veit J, Rainer G, Jaźwińska A. The zebrafish heart regenerates after cryoinjuryinduced myocardial infarction. BMC Dev Biol. 2011;11:21.

38. Schnabel K, Wu CC, Kurth T, Weidinger G. Regeneration of cryoinjury induced necrotic heart lesions in zebrafish is associated with epicardial activation and cardiomyocyte proliferation. PLoS One. 2011;6(4):e18503.

39. Sturzu AC, et al. The fetal mammalian heart generates a robust compensatory response to cell loss. Circulation. 2015;132(2):109-121.

40. Drenckhahn JD, et al. Compensatory growth of healthy cardiac cells in the presence of diseased cells restores tissue homeostasis during heart development. Dev Cell. 2008;15(4):521-533.

41. Porrello ER, et al. Transient regenerative potential of the neonatal mouse heart. Science. 2011;331(6020):1078-1080.

42. Porrello ER, et al. Regulation of neonatal and adult mammalian heart regeneration by the miR-15 family. Proc Natl Acad Sci U S A.
2013;110(1):187-192.

43. Lavine KJ, et al. Distinct macrophage lineages contribute to disparate patterns of cardiac recovery and remodeling in the neonatal and adult heart. Proc Natl Acad Sci U S A. 2014;111(45):16029-16034.

44. Polizzotti BD, Ganapathy B, Haubner BJ, Penninger JM, Kühn B. A cryoinjury model in neonatal mice for cardiac translational and regeneration research. Nat Protoc. 2016;11(3):542-552.

45. Kikuchi K, et al. Primary contribution to zebrafish heart regeneration by gata $4(+)$ cardiomyocytes. Nature. 2010;464(7288):601-605.

46. Jopling C, Sleep E, Raya M, Martí M, Raya A, Izpisúa Belmonte JC. Zebrafish heart regeneration occurs by cardiomyocyte dedifferentiation and proliferation. Nature. 2010;464(7288):606-609.

47. Boström P, et al. C/EBP $\beta$ controls exerciseinduced cardiac growth and protects against pathological cardiac remodeling. Cell. 2010;143(7):1072-1083.

48. Liu X, et al. miR-222 is necessary for exerciseinduced cardiac growth and protects against pathological cardiac remodeling. Cell Metab. 2015;21(4):584-595.

49. van Berlo JH, Maillet M, Molkentin JD. Signaling effectors underlying pathologic growth and remodeling of the heart. JClin Invest. 2013;123(1):37-45.

50. Hunter JJ, Chien KR. Signaling pathways for cardiac hypertrophy and failure. $N$ Engl JMed. 1999;341(17):1276-1283.

51. Kubin T, et al. Oncostatin M is a major mediator of cardiomyocyte dedifferentiation and remodeling. Cell Stem Cell. 2011;9(5):420-432.

52. Bisping E, et al. Gata4 is required for maintenance of postnatal cardiac function and protection from pressure overload-induced heart failure. Proc Natl Acad Sci U S A. 2006;103(39):14471-14476.

53. van Berlo JH, Aronow BJ, Molkentin JD. Parsing the roles of the transcription factors GATA-4 and GATA- 6 in the adult cardiac hypertrophic response. PLoS One. 2013;8(12):e84591.

54. Heineke J, et al. Cardiomyocyte GATA4 functions as a stress-responsive regulator of angiogenesis in the murine heart. JClin Invest. 2007;117(11):3198-3210.

55. Oka T, et al. Cardiac-specific deletion of Gata4 reveals its requirement for hypertrophy, compensation, and myocyte viability. Circ Res. 2006;98(6):837-845.

56. O'Donoghue M, Braunwald E. Natriuretic peptides in heart failure: should therapy be guided by BNP levels? Nat Rev Cardiol. 2010;7(1):13-20.

57. Lepilina A, et al. A dynamic epicardial injury response supports progenitor cell activity during zebrafish heart regeneration. Cell. 2006;127(3):607-619.

58. Gupta V, Gemberling M, Karra R, Rosenfeld GE, Evans T, Poss KD. An injury-responsive gata4 program shapes the zebrafish cardiac ventricle. Curr Biol. 2013;23(13):1221-1227.

59. Sallin P, de Preux Charles AS, Duruz V, Pfefferli C, Jaźwińska A. A dual epimorphic and compensatory mode of heart regeneration in zebrafish. Dev Biol. 2015;399(1):27-40.

60. Aguirre A, et al. In vivo activation of a conserved 
microRNA program induces mammalian heart regeneration. Cell Stem Cell. 2014;15(5):589-604.

61. Wu CC, et al. Spatially resolved genome-wide transcriptional profiling identifies BMP signaling as essential regulator of zebrafish cardiomyocyte regeneration. Dev Cell. 2016;36(1):36-49.

62. Fang Y, Gupta V, Karra R, Holdway JE, Kikuchi K, Poss KD. Translational profiling of cardiomyocytes identifies an early Jak1/ Stat 3 injury response required for zebrafish heart regeneration. Proc Natl Acad Sci U S A. 2013;110(33):13416-13421.

63. Jopling C, Suñe G, Morera C, Izpisua Belmonte JC. p38 $\alpha$ MAPK regulates myocardial regeneration in zebrafish. Cell Cycle. 2012;11(6):1195-1201.

64. Karra R, Knecht AK, Kikuchi K, Poss KD. Myocardial NF- $\mathrm{KB}$ activation is essential for zebrafish heart regeneration. Proc Natl Acad Sci U S A. 2015;112(43):13255-13260.

65. Kunisada K, Tone E, Fujio Y, Matsui H, YamauchiTakihara K, Kishimoto T. Activation of gp130 transduces hypertrophic signals via STAT3 in cardiac myocytes. Circulation. 1998;98(4):346-352.

66. Kunisada K, et al. Signal transducer and activator of transcription 3 in the heart transduces not only a hypertrophic signal but a protective signal against doxorubicin-induced cardiomyopathy. Proc Natl Acad Sci U S A. 2000;97(1):315-319.

67. Hirota $\mathrm{H}$, et al. Loss of a gp130 cardiac muscle cell survival pathway is a critical event in the onset of heart failure during biomechanical stress. Cell.1999;97(2):189-198.

68 . Jacoby JJ, et al. Cardiomyocyte-restricted knockout of STAT3 results in higher sensitivity to inflammation, cardiac fibrosis, and heart failure with advanced age. Proc Natl Acad Sci U S A. 2003;100(22):12929-12934.

69. Hilfiker-Kleiner D, et al. Signal transducer and activator of transcription 3 is required for myocardial capillary growth, control of interstitial matrix deposition, and heart protection from ischemic injury. Circ Res. 2004;95(2):187-195.

70. Maier HJ, et al. Cardiomyocyte-specific ІкB kinase (IKK)/NF- $\mathrm{kB}$ activation induces reversible inflammatory cardiomyopathy and heart failure. Proc Natl Acad Sci U S A. 2012;109(29):11794-11799.

71. Braz JC, et al. Targeted inhibition of p38 MAPK promotes hypertrophic cardiomyopathy through upregulation of calcineurin-NFAT signaling. JClin Invest. 2003;111(10):1475-1486.

72. Engel FB, Hsieh PC, Lee RT, Keating MT. FGF1/ p38 MAP kinase inhibitor therapy induces cardiomyocyte mitosis, reduces scarring, and rescues function after myocardial infarction. Proc Natl Acad Sci U S A. 2006;103(42):15546-15551.

73. Engel FB, et al. p38 MAP kinase inhibition enables proliferation of adult mammalian cardiomyocytes. Genes Dev. 2005;19(10):1175-1187.

74. Izumo S, Nadal-Ginard B, Mahdavi V. Protooncogene induction and reprogramming of cardiac gene expression produced by pressure overload. Proc Natl Acad Sci U S A. 1988;85(2):339-343.

75. Zhong W, et al. Hypertrophic growth in cardiac myocytes is mediated by Myc through a Cyclin D2-dependent pathway. EMBO J. 2006;25(16):3869-3879.

76. Li JM, Brooks G. Downregulation of cyclin- dependent kinase inhibitors $\mathrm{p} 21$ and $\mathrm{p} 27$ in pressure-overload hypertrophy. Am J Physiol. 1997;273(3 pt 2):H1358-H1367.

77. Hesse M, et al. Direct visualization of cell division using high-resolution imaging of M-phase of the cell cycle. Nat Commun. 2012;3:1076.

78. Hassink RJ, et al. Cardiomyocyte cell cycle activation improves cardiac function after myocardial infarction. Cardiovasc Res. 2008;78(1):18-25.

79. Pasumarthi KB, Nakajima H, Nakajima HO, Soonpaa MH, Field LJ. Targeted expression of cyclin D2 results in cardiomyocyte DNA synthesis and infarct regression in transgenic mice. Circ Res. 2005;96(1):110-118.

80. Woo YJ, et al. Therapeutic delivery of cyclin A2 induces myocardial regeneration and enhances cardiac function in ischemic heart failure. Circulation. 2006;114(1 suppl):I206-I213.

81. Cheng RK, et al. Cyclin A2 induces cardiac regeneration after myocardial infarction and prevents heart failure. Circ Res. 2007;100(12):1741-1748.

82. Shapiro SD, et al. Cyclin A2 induces cardiac regeneration after myocardial infarction through cytokinesis of adult cardiomyocytes. Sci Transl Med. 2014;6(224):224ra27.

83. Tane $\mathrm{S}$, et al. Repression of cyclin D1 expression is necessary for the maintenance of cell cycle exit in adult mammalian cardiomyocytes. J Biol Chem. 2014;289(26):18033-18044.

84. Sdek $\mathrm{P}$, et al. $\mathrm{Rb}$ and $\mathrm{p} 130$ control cell cycle gene silencing to maintain the postmitotic phenotype in cardiac myocytes. JCell Biol. 2011;194(3):407-423.

85. Chaudhry HW, et al. Cyclin A2 mediates cardiomyocyte mitosis in the postmitotic myocardium. J Biol Chem. 2004;279(34):35858-35866.

86. Jackson T, Allard MF, Sreenan CM, Doss LK, Bishop SP, Swain JL. The c-myc proto-oncogene regulates cardiac development in transgenic mice. Mol Cell Biol. 1990;10(7):3709-3716.

87. Xiao G, et al. Inducible activation of c-Myc in adult myocardium in vivo provokes cardiac myocyte hypertrophy and reactivation of DNA synthesis. Circ Res. 2001;89(12):1122-1129.

88. Trembley MA, Velasquez LS, de Mesy Bentley KL, Small EM. Myocardin-related transcription factors control the motility of epicardiumderived cells and the maturation of coronary vessels. Development. 2015;142(1):21-30.

89. Acharya A, et al. The bHLH transcription factor Tcf21 is required for lineage-specific EMT of cardiac fibroblast progenitors. Development. 2012;139(12):2139-2149.

90. Cai CL, et al. A myocardial lineage derives from Tbx18 epicardial cells. Nature. 2008;454(7200):104-108.

91. Zhou B, et al. Epicardial progenitors contribute to the cardiomyocyte lineage in the developing heart. Nature. 2008;454(7200):109-113.

92. Liu Q, et al. Epicardium-to-fat transition in injured heart. Cell Res. 2014;24(11):1367-1369.

93. Christoffels VM, Grieskamp T, Norden J, Mommersteeg MT, Rudat C, Kispert A. Tbx18 and the fate of epicardial progenitors. Nature. 2009;458(7240):E8-E9.

94. Rudat C, Kispert A. Wt1 and epicardial fate mapping. Circ Res. 2012;111(2):165-169.

95. Kikuchi K, et al. tcf $21^{+}$epicardial cells adopt non-myocardial fates during zebrafish heart development and regeneration. Development. 2011;138(14):2895-2902.

96. Lavine KJ, et al. Endocardial and epicardial derived FGF signals regulate myocardial proliferation and differentiation in vivo. Dev Cell. 2005;8(1):85-95.

97. Olivey HE, Svensson EC. Epicardial-myocardial signaling directing coronary vasculogenesis. Circ Res. 2010;106(5):818-832.

98. Zhou B, et al. Adult mouse epicardium modulates myocardial injury by secreting paracrine factors. JClin Invest. 2011;121(5):1894-1904.

99. Duan J, et al. Wnt1/ $\beta$-catenin injury response activates the epicardium and cardiac fibroblasts to promote cardiac repair. $E M B O J$. 2012;31(2):429-442.

100.Ruiz-Villalba A, et al. Interacting resident epicardium-derived fibroblasts and recruited bone marrow cells form myocardial infarction scar. JAm Coll Cardiol. 2015;65(19):2057-2066.

101.Wang J, Cao J, Dickson AL, Poss KD. Epicardial regeneration is guided by cardiac outflow tract and Hedgehog signalling. Nature. 2015;522(7555):226-230.

102. González-Rosa JM, Peralta M, Mercader N. Panepicardial lineage tracing reveals that epicardium derived cells give rise to myofibroblasts and perivascular cells during zebrafish heart regeneration. Dev Biol. 2012;370(2):173-186.

103. Wang J, Cao J, Dickson AL, Poss KD. Epicardial regeneration is guided by cardiac outflow tract and Hedgehog signalling. Nature. 2015;522(7555):226-230.

104.Kikuchi K, et al. Retinoic acid production by endocardium and epicardium is an injury response essential for zebrafish heart regeneration. Dev Cell. 2011;20(3):397-404.

105. Marín-Juez R, et al. Fast revascularization of the injured area is essential to support zebrafish heart regeneration. Proc Natl Acad Sci U S A. 2016;113(40):11237-11242.

106.Zhao L, et al. Notch signaling regulates cardiomyocyte proliferation during zebrafish heart regeneration. Proc Natl Acad Sci U S A. 2014;111(4):1403-1408.

107. Harrison MR, et al. Chemokine-guided angiogenesis directs coronary vasculature formation in zebrafish. Dev Cell. 2015;33(4):442-454.

108. Hulsmans M, Sam F, Nahrendorf M. Monocyte and macrophage contributions to cardiac remodeling. J Mol Cell Cardiol. 2016;93:149-155.

109. Li L, Yan B, Shi YQ, Zhang WQ, Wen ZL. Live imaging reveals differing roles of macrophages and neutrophils during zebrafish tail fin regeneration. J Biol Chem. 2012;287(30):25353-25360.

110. Petrie TA, Strand NS, Yang CT, Tsung-Yang C, Rabinowitz JS, Moon RT. Macrophages modulate adult zebrafish tail fin regeneration. Development. 2014;141(13):2581-2591.

111. Aurora AB, et al. Macrophages are required for neonatal heart regeneration. JClin Invest. 2014;124(3):1382-1392.

112. Singer $M$. The influence of the nerve in regeneration of the amphibian extremity. QRev Biol. 1952;27(2):169-200.

113. Mahmoud AI, et al. Nerves regulate cardiomyocyte proliferation and heart regeneration. Dev Cell. 2015;34(4):387-399. 
114. White IA, Gordon J, Balkan W, Hare JM. Sympathetic reinnervation is required for mammalian cardiac regeneration. Circ Res. 2015;117(12):990-994.

115. Santini MP, Forte E, Harvey RP, Kovacic JC. Developmental origin and lineage plasticity of endogenous cardiac stem cells. Development. 2016;143(8):1242-1258.

116. Hsieh PC, et al. Evidence from a genetic fatemapping study that stem cells refresh adult mammalian cardiomyocytes after injury. Nat Med. 2007;13(8):970-974.

117. van Berlo JH, et al. c-kit ${ }^{+}$cells minimally contribute cardiomyocytes to the heart. Nature. 2014;509(7500):337-341.

118. Liu Q, et al. Genetic lineage tracing identifies in situ Kit-expressing cardiomyocytes. Cell Res. 2016;26(1):119-130.

119. Sultana N, et al. Resident c-kit(+) cells in the heart are not cardiac stem cells. Nat Commun 2015;6:8701.

120. Uchida S, et al. Sca1-derived cells are a source of myocardial renewal in the murine adult heart. Stem Cell Reports. 2013;1(5):397-410.

121. Lin Z, Pu WT. Strategies for cardiac regeneration and repair. Sci Transl Med.2014;6(239):239rv1.

122. Morikawa Y, et al. Actin cytoskeletal remodeling with protrusion formation is essential for heart regeneration in Hippo-deficient mice. Sci Signal. 2015;8(375):ra41.

123. Tian Y, et al. A microRNA-Hippo pathway that promotes cardiomyocyte proliferation and cardiac regeneration in mice. Sci Transl Med. 2015;7(279):279ra38.

124. Li J, et al. Alpha-catenins control cardiomyocyte proliferation by regulating Yap activity. Circ Res. 2015;116(1):70-79.

125. Heallen T, et al. Hippo pathway inhibits Wnt signaling to restrain cardiomyocyte proliferation and heart size. Science. 2011;332(6028):458-461.

126.von Gise A, et al. YAP1, the nuclear target of Hippo signaling, stimulates heart growth through cardiomyocyte proliferation but not hypertrophy. Proc Natl Acad Sci U S A. 2012;109(7):2394-2399.

127. Xin M, et al. Regulation of insulin-like growth factor signaling by Yap governs cardiomyocyte proliferation and embryonic heart size. Sci Signal. 2011;4(196):ra70.
128.Xin M, et al. Hippo pathway effector Yap promotes cardiac regeneration. Proc Natl Acad Sci U S A. 2013;110(34):13839-13844.

129. Heallen T, et al. Hippo signaling impedes adult heart regeneration. Development. 2013;140(23):4683-4690.

130. Lin Z, et al. Cardiac-specific YAP activation improves cardiac function and survival in an experimental murine MI model. Circ Res. 2014;115(3):354-363.

131. Lin Z, et al. Acetylation of VGLL4 regulates HippoYAP signaling and postnatal cardiac growth. Dev Cell. 2016;39(4):466-479.

132. Tao G, et al. Pitx2 promotes heart repair by activating the antioxidant response after cardiac injury. Nature. 2016;534(7605):119-123.

133. Yu L, Daniels JP, Wu H, Wolf MJ. Cardiac hypertrophy induced by active Raf depends on Yorkie-mediated transcription. Sci Signal. 2015;8(362):ra13.

134. Wackerhage H, Del Re DP, Judson RN, Sudol M, Sadoshima J. The Hippo signal transduction network in skeletal and cardiac muscle. Sci Signal. 2014;7(337):re4.

135. Del Re DP, et al. Yes-associated protein isoform 1 (Yap1) promotes cardiomyocyte survival and growth to protect against myocardial ischemic injury. J Biol Chem. 2013;288(6):3977-3988.

136. Brockes JP, Lemke GE, Balzer DR. Purification and preliminary characterization of a glial growth factor from the bovine pituitary. J Biol Chem. 1980;255(18):8374-8377.

137. Holmes WE, et al. Identification of heregulin, a specific activator of p185erbB2. Science. 1992;256(5060):1205-1210.

138. Meyer D, Birchmeier C. Multiple essential functions of neuregulin in development. Nature. 1995;378(6555):386-390.

139. Gassmann M, et al. Aberrant neural and cardia development in mice lacking the ErbB4 neuregulin receptor. Nature. 1995;378(6555):390-394.

140.Lee KF, Simon H, Chen H, Bates B, Hung MC, Hauser C. Requirement for neuregulin receptor erbB2 in neural and cardiac development. Nature. 1995;378(6555):394-398.

141. Bersell K, Arab S, Haring B, Kühn B. Neuregulin1/ErbB4 signaling induces cardiomyocyte proliferation and repair of heart injury. Cell.
2009;138(2):257-270.

142. Polizzotti BD, et al. Neuregulin stimulation of cardiomyocyte regeneration in mice and human myocardium reveals a therapeutic window. $S c i$ Transl Med. 2015;7(281):281ra45.

143. Grego-Bessa J, et al. Notch signaling is essential for ventricular chamber development. Dev Cell. 2007;12(3):415-429.

144.Gemberling M, Karra R, Dickson AL, Poss KD. Nrg1 is an injury-induced cardiomyocyte mitogen for the endogenous heart regeneration program in zebrafish. Elife. 2015;4:e05871.

145. D'Uva G, et al. ERBB2 triggers mammalian heart regeneration by promoting cardiomyocyte dedifferentiation and proliferation. Nat Cell Biol. 2015;17(5):627-638.

146.Wei K, et al. Epicardial FSTL1 reconstitution regenerates the adult mammalian heart. Nature. 2015;525(7570):479-485.

147. Porrello ER, et al. MiR-15 family regulates postnatal mitotic arrest of cardiomyocytes. Circ Res. 2011;109(6):670-679.

148. Eulalio A, et al. Functional screening identifies miRNAs inducing cardiac regeneration. Nature. 2012;492(7429):376-381

149. Nakada Y, et al. Hypoxia induces heart regeneration in adult mice. Nature. 2017;541:222-227.

150. Velazquez EJ, et al. Coronary-artery bypass surgery in patients with ischemic cardiomyopathy. N Engl J Med. 2016;374(16):1511-1520.

151. Sadek HA, et al. Multi-investigator letter on reproducibility of neonatal heart regeneration following apical resection. Stem Cell Reports. 2014;3(1):1.

152. Andersen DC, Ganesalingam S, Jensen $\mathrm{CH}$, Sheikh SP. Do neonatal mouse hearts regenerate following heart apex resection? Stem Cell Reports. 2014;2(4):406-413.

153. Darehzereshki A, et al. Differential regenerative capacity of neonatal mouse hearts after cryoinjury. Dev Biol. 2015;399(1):91-99.

154.Laflamme MA, Murry CE. Heart regeneration. Nature. 2011;473(7347):326-335.

155. Ali SR, Hippenmeyer S, Saadat LV, Luo L, Weissman IL, Ardehali R. Existing cardiomyocytes generate cardiomyocytes at a low rate after birth in mice. Proc Natl Acad Sci US A. 2014;111(24):8850-8855. 